\title{
Novel technique of controlled laser air-force detection for rheological properties of polymers
}

\author{
Hubo $\mathrm{Xu}^{1,2}$, Runzhi Hu${ }^{1}$, Yingzi Lin ${ }^{3}$, Hincapie Juan ${ }^{3}$, Xiuying Tang ${ }^{1 *}$ \\ (1. College of Engineering, China Agricultural University, Beijing 100083, China; \\ 2. Health Science Center, Peking University, Beijing 100191, China; \\ 3. College of Engineering, Northeastern University, MA 02115, USA)
}

\begin{abstract}
A novel controlled laser air-force detection (CLAFD) technique was developed to detect the rheological properties of polymers with the characteristics of non-destruction and cross-contamination free. Dynamic testing and static testing were carried out in the technique. Back propagation neural network algorithm was used to establish the air-force control model. The replicability of CLAFD system was analyzed, the viscoelastic properties of polyurethane were investigated using alternating load testing. A comparative analysis of performances was made between the CLAFD and the texture analysis (TA) on the testing of creep-recovery and stress relaxation. The results demonstrated that the CLAFD system had good replicability. The lagging phase angle was between $0^{\circ}-90^{\circ}$ in the testing of alternating load. This illustrated that the CLAFD technique could be used to detect viscoelasticity. The parameters of response speed and the precision of the CLAFD entirely surpassed the TA on the creep-recovery testing. The CLAFD technique will provide a new real-time, non-destruction and cross-contamination-free detection method for material science, especially for those materials such as artificial biological tissue and function food products.
\end{abstract}

Keywords: controlled laser air-force detection (CLAFD) technique, biological tissues, rheological properties, statics analysis, dynamic analysis

DOI: $10.25165 /$ j.ijabe.20221501.6494

Citation: Xu H B, Hu R Z, Lin Y Z, Juan H, Tang X Y. Novel technique of controlled laser air-force detection for rheological properties of polymers. Int J Agric \& Biol Eng, 2022; 15(1): 62-70.

\section{Introduction}

As one of the four major components in material science, polymers are widely used in various fields of industry. It is of great significance to conduct a thorough and systematic study on the rheological properties of different forms of polymers ${ }^{[1-5]}$. On the other hand, there are limitations from the sampling complexity and the ethics to prepare the samples when studying the properties of biological organization. It is easier to build a biological polymer sample with similar mechanical properties, than it would be to build based on biological organization. Using polyurethane materials and adjusting their formulation can be used to modify the biological organization of material. The use of polymer materials to simulate biological tissues is an important research method in life science ${ }^{[6,7]}$.

Mechanical properties are basic properties of the structure material and the function material. The static and dynamic analyses provide important means to analyze the material properties under different conditions ${ }^{[8-10]}$. Many of the materials coming from the chemical and agricultural industries are polymers and most of them are viscoelastic. Several instruments were

Received date: 2021-01-31 Accepted date: 2021-12-03

Biographies: Hubo Xu, PhD, Assistant Research Fellow, research interest: neuromorphic computing and intelligent detection technique, Email: hubo_xu@bjmu.edu.cn; Runzhi Hu, MD, research interest: artificial intelligence technology, Email: runzhi.hu@polyu.edu.hk; Yingzi Lin, PhD, Professor, research interest: human-machine systems, Email: yi.lin@northeastern.edu; Hincapie Juan, MD, research interest: human-machine systems, Email: j.hincapie@northeastern.edu.

*Corresponding author: Xiuying Tang, $\mathrm{PhD}$, Professor, research interest: intelligent detection technique, Email: txying@cau.edu.cn. developed to conduct the static/dynamic testing of the polymer, such as the universal testing machine, the texture analyzer, and the dynamic thermomechanical analysis, etc. Testing of these methods is relatively stable. The signal of excitation and acquisition is carried out through mechanics. These methods provide stable testing, however, with shortcomings. Test efficiency is affected by the mechanical structure, because the probe must be disassembled and re-assembled in every single experiment. Cross-Contamination between samples may occur in some mechanical testing processes of biomaterials. The hard metal probe may damage brittle and hard polymer materials. Therefore, these methods could not perform non-destructive detection. These methods have been used for many years; there is a clear need for the development of new technologies in the field of rheological properties detection of solid polymers.

Pressia et al. ${ }^{[11]}$ first put forward a method with a combination of the fluid impulse jet and the laser radiation beam to detect the firmness of fruits nondestructively. Later it was known as the laser air-puff (LAP) ${ }^{[12]}$. Lee et al. ${ }^{[13]}$ used the LAP technique to detect the tenderness of chicken meat. In this study, the air-puff was applied to compress chicken samples transported by a conveyor belt. The laser displacement sensor was used to scan the deformation of samples. The parameters calculated from the deformation profiles were used to predict chicken tenderness. This study showed that LAP could potentially be implemented as an online tool. The sugar-based and fat-based materials were chosen as samples to study their viscosity by Morren et $\mathrm{al}^{[14]}$. They found that the data of the LAP was correlated to the viscosity. Similarly, Long et al. ${ }^{[15]}$ built a laser-air-force beef tenderness detection system. The sample was impacted by air-force to produce impact deformation, and then the characteristic parameters 
of the deformation curve were extracted. Finally, the beef tenderness was predicted. The correlation coefficient of the prediction model was 0.84 . The freshness of beef was predicted by the same LAP testing device ${ }^{[16,17]}$. The correlation coefficient of freshness prediction was less than 0.9 as well. Although the tenderness and freshness are qualities of meat, the "bridge" that connects the deformation curve to these qualities is the properties of viscoelasticity ${ }^{[18]}$. This means that the LAP has application potential in the property detection of food materials. However, the prediction accuracy was low. Maclone and Jordan ${ }^{[19]}$ even suggested that the technique could be only used for coarse screening. They basically denied the possibilities of precise classification and quantitative detection.

According to the previous studies ${ }^{[16-18]}$, it was discovered that at different distances from the nozzle, the air-force is different. The relationship between the air-force and the distance is non-linear. This means that as the sample is being loaded by air-force, the force on its surface is constantly changing in a way that is difficult to identify. Meanwhile, the height and shape of different samples are not completely the same, increasing the uncontrollability of the excitation signals. The further analysis concludes that the LAP technique has problems with the divergence of the air, creating a "concave surface". Because the curvature of the surface is constantly changing, the laser is not targeting the same point during the experiment. The force on different points on the curved surface is different, and varies with time. This is another fundamental problem with the LAP technique. Because of the existing problems, the LAP technique could not be used in actual production. This is why in the past twenty years, studies about LAP have not produced satisfying results, although the research objects constantly changed. In general, LAP is still in the stage of conception verification.

To solve the problems above, a novel controlled laser air-force detection (CLAFD) system was developed in this study. The control processing of the signal, was comprised of the laser signal, the pressure signal, and the air-force signal. This illustrates the complexity of the development of the control system. The cores of the control of the laser air-force technology were in the controls of the laser range and the air-force regulation. The air-force generation system should be practically closed in the design of the structure of the system. This is the key to realizing pressure control. Keeping the paths of the laser beam and gas coaxial is the key to ensuring the accurate acquisition of displacement information. The purpose of air pressure control is to control the air-force on the surface of the sample. Therefore, accurate control of the air-force on the surface of the sample is the key to the high-precision detection of the system. However, when the air-force is injected into an open-source environment, it will attenuate due to the effect of air resistance. This leads to a non-linear relationship among the pressure, the air-force, and the distance. Multivariate nonlinear regression and neural network algorithms are important modeling methods. Torabi-Kaveh et al. ${ }^{[20]}$ pointed out that the latter has more advantages than the former.

The specific design of the loading signals included: dynamic loading and static loading signals. The transient and alternating signals are normally used for dynamic loading. The transient testing is used to detect the response characteristics of polymer after instantaneous impact. It has the characteristics of high detection efficiency and the great significance of online detection. This is an important supplement of laser air-puff technology compared to traditional detection methods. The alternating load signal is of great significance to the identification of viscoelastic properties and fatigue strength test of polymer materials. In the static test, the creep-recovery and stress relaxation testing are important means to analyze the rheological properties of polymer materials, which are of great significance to the quality testing of materials. Polyurethane, which is widely used in industrial fields was the sample. The objectives of this research were 1) the creative and reasonable structure of CLAFD system was designed, the air-force and pressure control models were established by neural network algorithm; 2) the embedded technology was used to develop the software system under Linux. Four testing functions of the CLAFD system were developed; 3) the repeatability of CLAFD system was analyzed by transient testing. The mechanical properties of polyurethane were analyzed by the alternating load testing; 4) the excitation control signals of the creep-recovery and the stress relaxation were compared between the CLAFD and the classic method-the texture analysis (TA).

\section{Development of the CLAFD system}

\subsection{Hardware structure}

The structure of CLAFD system was designed, according to the requirements of the laser air-force detection method and the realization of the control function of CLAFD system on the detection of polymer rheological properties. As shown in Figure 1 , the structure of CLAFD system mainly included the body, lifting system, air-force generation system, deformation detection system, stress sensing system, and control and information processing system. The lifting system was composed of a stepping motor driver (HST2802A-H, Beijing HST Technology Co., Ltd, China), a stepping motor (KSAV1010/2030-ZF, ZOLIX Instruments Co., Ltd, China), and a lifting platform (Customization, Beijing HST Technology Co., Ltd, China). It provided the support for samples and it could adjust the position of the sample in the vertical direction. The air-force generating system was installed on the upper part of the body. The system included an air compressor (4X550W-120L, Autus Industry and Trade Co., Ltd, China; independent part, is not shown in Figure 1), pressure maintaining valve (P98020-501-3, SMC Co., Ltd, Japan), two-stage air filter (AC30C-03DG-A, SMC Co., Ltd; ensuring that air is clean), electrical proportional valve (ITV2030-21F2L, SMC Co., Ltd, Japan), solenoid valve (VX21, SMC Co., Ltd, Japan), and air chamber (self-made). The function of the system was to realize the generation and regulation of high-pressure air force. From top to bottom, the air chambers include the quartz window, body, and quartz micro-nozzle. The deformation detection system included a laser displacement sensor (IL100, KEYENCE Co., Ltd, Japan), signal amplifier (IL1000, KEYENCE Co., Ltd., Japan), and signal conversion module (DL-RSIA, KEYENCE Co., Ltd., Japan). The laser displacement sensor was located directly above the air chamber and was connected with the signal amplifier, and it was connected with the laser signal conversion module. The laser displacement sensor was for real-time acquisition of deformation information of samples. The incident beam of the laser displacement sensor was coaxial with the outlet of the nozzle. The stress sensing system included the electromagnetic force balance sensor (Customization, Shanghai Sunny Hengping Scientific Instrument Co., Ltd, China) and its conversion module. The electromagnetic force balance sensor was mounted on top of the lifting platform. A tray was arranged on the electromagnetic force balance sensor, and the tray was used for placing samples. 
The sample was placed below the nozzle. The electromagnetic force balance sensor could acquire real-time air-force information of the sample surface which is planar sensing. The resolution of the electromagnetic force balance sensor was $0.001 \mathrm{~N}$ and the resolution matches the stress resolution requirement of creep-recovery and stress relaxation.

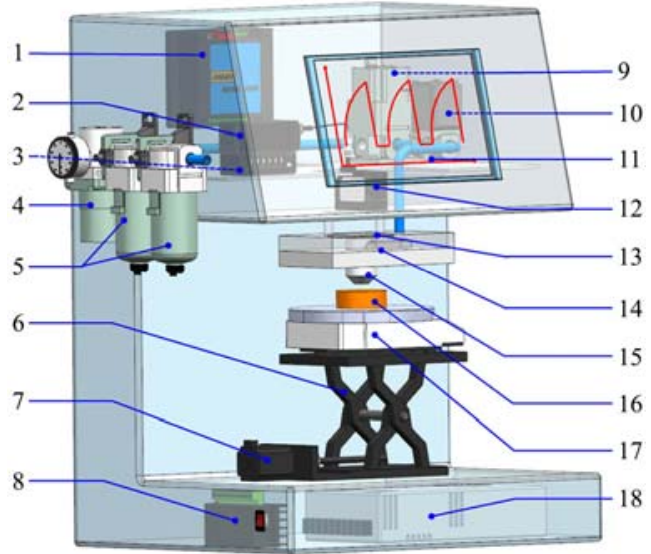

1. D/A conversion module for electric proportional valve 2. Laser signal amplifier 3. A/D conversion module of laser displacement sensor 4. Pressure maintaining valve 5. Two-grade air filter 6. Lifting platform 7. Stepping motor 8. Stepping motor driver 9. Electric proportional valve 10. Solenoid valve 11. Embedded integrative machine 12. Laser displacement sensor 13. Quartz window 14. Air chamber body 15. Quartz micro-nozzle 16. Sample 17. Electromagnetic force balance sensor 18. Regulated power supply

Figure 1 Schematic of controlled laser air-force detection system

The control and information processing system included the embedded microprocessor (Cortex-A8, ARM Co., Ltd.), the embedded integrated machine (LJD-LINUX-LH070T, BOMC Co., Ltd.) with touch screen, and the analog-to-digital converter. The embedded integrative machine was connected to the embedded microprocessor. The functions were developed in the embedded integrative computer in which, the parameters setting, command triggering soft keyboard and data graphics display. The embedded microprocessor received the command from the trigger button and controlled the solenoid valve, electric proportional valve and motor driver of the elevator, etc. The embedded microprocessor stored and processed the received data, and displayed the information of deformation in the data graphics display area. The pressure gauge on the electric proportional valve was used to adjust and display the pressure of the electric proportional valve. The control voltage range of the electric proportional valve was $0-3 \mathrm{~V}$, and the air flux of the electric proportional valve increased gradually. The pressure of the output of the electric proportional valve increased gradually from $0 \mathrm{MPa}$ to $0.3 \mathrm{MPa}$.

The materials of window and nozzle were quartz with $90 \%$ transmittance. The processing method of windows and nozzle was a combination of mechanical processing and flame polishing. According to the processing ability and the requirement of the function on quartz material, the internal mechanism of micro-nozzle was a slender hole with $3 \mathrm{~mm}$ inner diameter and $9 \mathrm{~mm}$ length. The material and structure of the nozzle ensured that the optical path produced by the laser displacement sensor was coaxial with the air path ejected from the nozzle. This ensured the accurate acquisition of deformation information of the sample and as shown in Figure 2, this also ensured a good convergence of the air column emitted by the nozzle. In this study, the diameter of the intake pipe of the air chamber was $15 \mathrm{~mm}$, which was much larger than the inner diameter of the nozzle $(3 \mathrm{~mm})$. This created an approximately closed environment of the air-force system and achieved effective pressure control of the pressure by the electric proportional valve.

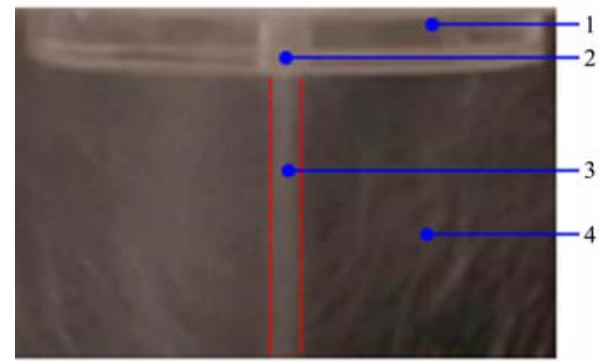

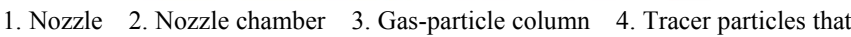
bounce back after touching the sample

Figure 2 Particle tracing of nozzle jet

\subsection{Establishment of air-force excitation signal control model}

Effective control of air-force excitation signal was the key to realize the detection of the rheological properties of polymer. The influencing factors of the air-force $(F)$ on the surface of the sample included the distance $(D)$ acquired by the laser displacement sensor and the output pressure $(P)$ of the electric proportional valve. A multi-level test of $D$ and $P$ factors should be conducted to acquire the basic data for the control model construction. The electromagnetic force balance sensor ranged from $6 \mathrm{~mm}$ from the nozzle to $50 \mathrm{~mm}$, which is the maximum range of laser displacement sensor. The distance interval was $1 \mathrm{~mm}$, totaling 45 levels. The pressure of the electric proportional valve was adjusted from 0.01 to $0.3 \mathrm{MPa}$, and the interval was $0.01 \mathrm{MPa}$, totaling 30 levels. The electromagnetic force balance sensor was used to obtain the air-force data corresponding to the specific pressure at each distance. The sampling time at each level was 20 seconds and the sampling frequency was 50 times per second. In order to avoid the influence of the rising stage of increasing pressure, the average value of $10-20 \mathrm{~s}$ data was taken as the air-force value under the specific factors and levels. Therefore, a total of 1380 data values were obtained and used to establish the air-force excitation signal control model. The relationship among those three parameters F, P, and D is shown in Figure 3.

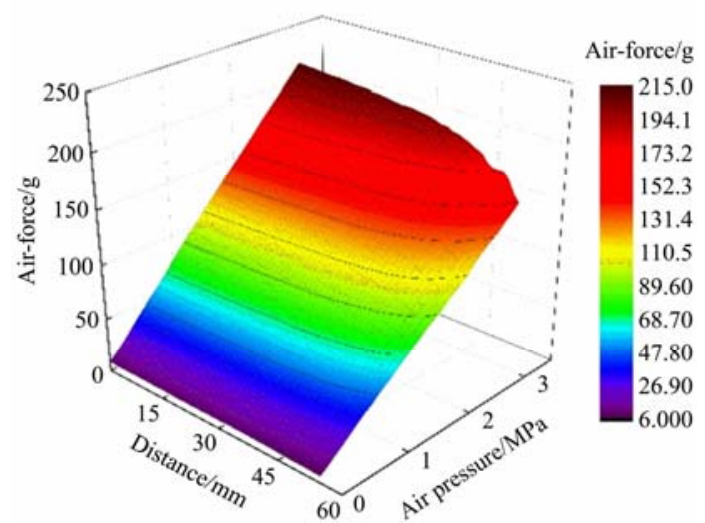

Figure 3 The relationship among three parameters $F, P$, and $D$

The BP neural network was a method to store and process non-linear information by using network topology and weight distribution. It had high parallelism and could conduct complex data calculation ${ }^{[21]}$. The amount of data used in this study was large, and the relationship among F, P, and D were non-linear (Figure 3). Therefore, the BP neural network algorithm was used to establish the air-force excitation signal regulation model in this study. Meanwhile, the neural network algorithm was implemented in Matlab R2016b (Mathworks Inc., Natick, MA). 
The basic topological structure of the neural network consists of the input layer, hidden layer, and output layer. Each layer consists of neurons and each neuron includes weights (W), biases (B), adders and activation functions. In this study, the Levenberg-Marquardt algorithm was used as a back-propagation training algorithm to determine the weights and biases of each neuron. The Sigmoid function was used as the excitation function of neuron hidden layer, and the Pureline function was used as the activation function of the output layer. The output of the neural network could only be calculated by input through multiple neurons, but it could not get the input from the output. Therefore, according to the control function for the testing of the rheological property of polymer, $\mathrm{P}$ and the $\mathrm{D}$ were needed to predict the $F$, but also $P$ should be deduced from $F$ and $D$.

The number of hidden layer neuron nodes directly affects the effectiveness of the neural network algorithm in modeling. There are two methods to determine the number of neurons: the empirical formula method and the trial-and-error method. In this study, the number of neurons was determined by combining the two methods. The empirical equation is as follows:

$$
n^{*}=\sqrt{n+m}+a
$$

where, $n^{*}$ is the number of hidden layer neuron nodes; $n$ is the number of input; $m$ is the number of output; $a$ is the number of integers in $[1,10]$.

There were two inputs and one output in the network in this study, according to Equation (1), the number of hidden layer neuron nodes was 3-13. In theory, the more neurons in the hidden layer, the stronger the processing ability of the neural network is, but this would also cause problems such as data processing speed reduction and model instability. Therefore, the performance of models established by 3 to 20 hidden neurons was compared in this study. The Pearson correlation coefficient was close to 1 for both air-force and pressure prediction processes, which showed that the neural network algorithm had a good modeling ability and forecasting effect. In general, when the number of neurons in the hidden layer increased, the root mean square error (RMSE) showed a downward trend. For all the sets, the training set, calibration set, and verification set, the model showed the smaller RMSE when the number of neurons was 12,13,14,17, 18 and 19 for air-force prediction. The model showed the smaller RMSE when the number of neurons was $11,12,16,17,18$ and 20 for pressure prediction. According to the result of Equation (1), when the number of neurons was within 3-13, the model was relatively stable. Therefore, the number of neurons in the hidden layer was determined to be 12 in this study. At this point, the Pearson correlation coefficients of the calibration set, the prediction set, and the test set were $0.999972,1.000000$ and 1.000000 respectively. The RMSE were $0.186585 \mathrm{~g}, 0.216310 \mathrm{~g}$, and $0.213976 \mathrm{~g}$, respectively for the air-force prediction. For the pressure prediction, the Pearson correlation coefficients of the correction set, prediction set, and test set were $0.999966,0.999999$ and 1.000000 with the RMSE of $0.500882 \times 10^{-6} \mathrm{~g}, \quad 0.743195 \times 10^{-6} \mathrm{~g}$, $0.580712 \times 10^{-6} \mathrm{~g}$, respectively.

From the results above, it could be observed that the RMSE of the air-force prediction model was approximately $0.2 \mathrm{~g}$, and the RMSE of the pressure prediction model was approximately $0 \mathrm{~g}$. The Pearson correlation coefficient of the controlling model was approximately 1 among air-force, the pressure and the distance for both of them. This demonstrated that the air-force controlling model established by the BP neural network had high controlling precision and could be used for air-force regulation of the CLAFD system.

\subsection{Software development}

The Cortex A8 embedded microprocessor was used in the CLAFD system. The operating environment of the software was Linux. Compared to the one-chip control, the embedded control could flexibly use multi-threads to write control programs. Qt widgets for technical applications (QWT) and C++ programming language were used in developing the software interface in this research. The deformation of samples was displayed in real-time through the interface. This software system mainly included the encapsulation of the foundation class of the control system program, the compilation of control system program, and the design of software operation interfaces.

As shown in Figure 4, the components of the software system interfaces of CLAFD technique mainly included the creep-recovery detection interface, the stress relaxation detection interface, the transient testing interface, and the alternating load testing interface. Every single operating interface consisted of four parts: the title bar located at the top of the interface, the plot area of sample material deformation curve located at the middle of the interface, the
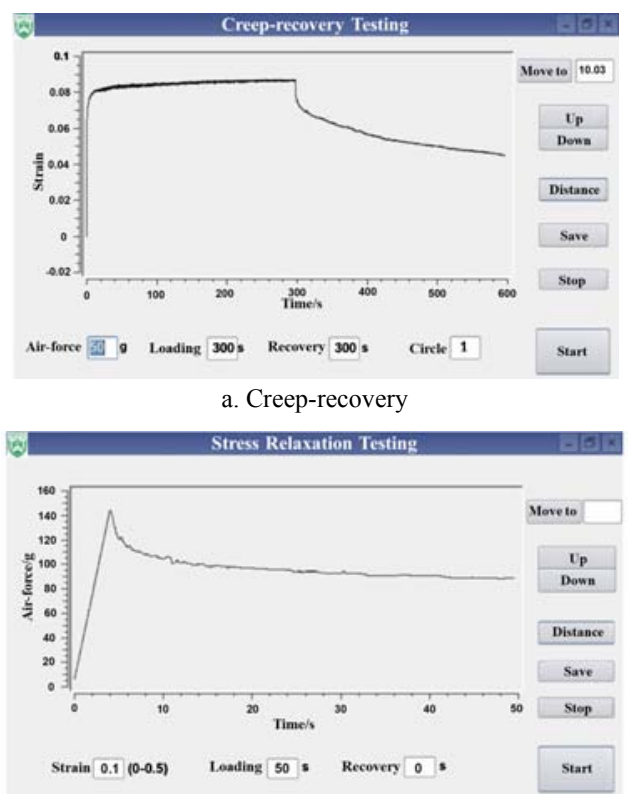

b. Stress relaxation

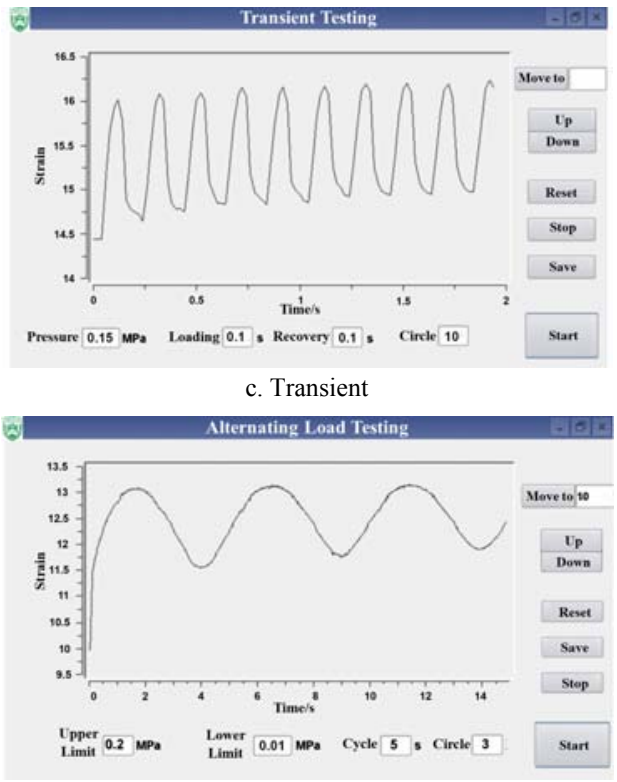

d. Alternating load

Figure 4 Different function software interfaces 
parameters setting area located at the bottom of the interface and the control area of executing parts located at the right of the interface. The hardware control, data processing and real-time display of the CLAFD technique were carried out by using the software.

\section{Materials and methods}

\subsection{Polyurethane sample}

In this study, a cast polyurethane material, called the Hei-Cast 8400 (H\&K Ltd, Tokyo, Japan), was selected to prepare the patterned samples. The Hei-Cast 8400 is a typical casting material, which includes Components A (polymer polyol), B (polyisocyanate), and $\mathrm{C}$ (heterocyclic diol, as chain extender). The low viscosity of the $\mathrm{A}, \mathrm{B}$, and $\mathrm{C}$ mixture promises a fine flowing property and exact shape replication. Once the mixture is cured, the sample is highly elastic and hard to tear. By controlling the content of $\mathrm{C}$, the Shore hardness can be adjusted from $10 \mathrm{HA}$ to $90 \mathrm{HA}$. In this study, the mass ratios of $\mathrm{A}, \mathrm{B}$, and $\mathrm{D}$ were set to 100:100:600, 100:100:500, 100:100:400, 100:100:300, 100:100:200, 100:100:150 and the mixture was cured under $60^{\circ} \mathrm{C}$ for $90 \mathrm{~min}$. The Shore hardness of the samples is about 10, 20, 30, 40, 50, and 60 HA. 20 samples for every single Shore hardness, therefore, there were 120 samples prepared for the study. The special model was used to produce the sample, and the geometrical sizes of samples are $\varnothing(23.89 \pm 0.60) \mathrm{mm}$ (diameter) $\times(13.91 \pm 0.10) \mathrm{mm}$ (height).

\subsection{Rheological property detection based on CLAFD technique}

In the study, the parameters setting of the different rheological property detection modules of the CLAFD system were as follows: for the transient testing, the setting value of the air-force was $0.2 \mathrm{MPa}$ and the loading time was $1 \mathrm{~s}$. In order to ensure that the polyurethane material could be recovered after one compression, the recovery time was set to $6 \mathrm{~s}$, the transient excitation was pulsed 5 times on every single cyclic test. Each sample was loaded 5 times. On this basis, three evaluation parameters, peak error, peak time error and excitation energy (the area enclosed by the curve and the time axis of $y=0$ ) error, were proposed to evaluate the repeatability of CLAFD system.

For the alternating load testing, the upper limit of loading was $0.2 \mathrm{MPa}$, the lower limit was $0.02 \mathrm{MPa}$, and the sampling period was $10 \mathrm{~s}$. For the creep-recovery test, the loading force was $40 \mathrm{~g}$, the loading time was $30 \mathrm{~s}$, the unloading force was $0 \mathrm{~g}$, and the unloading time was $30 \mathrm{~s}$. For the stress relaxation test, the strain was 0.02 and the loading time was $60 \mathrm{~s}$. The number of polyurethane samples was 120 for every test module.

\subsection{Rheological property detection based on texture analyzer} (TA)

Texture analyzer is an important instrument for mechanical and rheological properties analysis of materials in the food and chemical industry. The texture analyzer (TA. XT Plus, Texture Technologies Corp., and Stable Micro Systems. Ltd.) was used as the comparative analysis object of the CLAFD system control performance in this study. Due to the functional limitation of the TA and the properties of polyurethane materials, the application of texture mechanical analysis in transient and sinusoidal characteristics tests had predictable limitations. Therefore, in this study, the creep-recovery test and the stress relaxation test were carried out using TA to compare the control performance with CLAFD technique. The test process and parameters setting of the two modules were uniform for the CLAFD technique. The probe was spherical (the diameter was $1.27 \mathrm{~cm}$ ), the trigger force was $5 \mathrm{~g}$, and the travel speed was $0.5 \mathrm{~mm} / \mathrm{s}$.

\subsection{Control performance evaluation index of CLAFD technique and TA technique}

In this study, the following parameters were proposed for evaluating the control performance of the creeping stage and stress relaxation loading signals: the rise time $\tau_{r}(\mathrm{~s})$ : the time required to rise from zero to the first time that the force reaches the steady-state value of loading after the system triggered; the peak time $\tau_{p}(\mathrm{~s})$ : the time required to reach the first peak after the force exceeds the steady-state value under loading; the settling time $\tau_{s}(\mathrm{~s})$ the shortest time required for the force to reach and maintain within the error band $( \pm 2 \%)$ of the steady loading value; the delay time $\tau_{d}$ (s): the time required to reach $50 \%$ of the loading stability for the first time; the peak overshoot $\sigma_{p}(\%)$ : the percentage of air-force peak value exceeding the steady value of loading. The error band $\pm \Delta(\mathrm{g})$ : fluctuation of air-force after the system reaches a steadystate; the steady-state overshoot $\sigma(\%)$ : the percentage of steady-state loading value exceeding the set value.

For the test of creep-recovery, the stage of recovery is the process of material recovery after the constant loading. The evaluation parameters were similar to the loading stage: the rise time $\tau_{r}^{\prime}(\mathrm{s})$ : the time required for the force to descend from the last moment of the loading steady-state to the first time it reaches the steady value of unloading steady-state; the peak time $\tau_{p}^{\prime}$ (s): the time required to reach the first valley after the force exceeds the steady-state value of unloading; the settling time $\tau_{s}^{\prime}(\mathrm{s})$ : the shortest time required for the force to reach and maintain within the $\pm 2 \%$ error band of the steady-state value of unloading; the delay time $\tau_{d}^{\prime}$ (s): the time it takes for the first air-force to reach $50 \%$ of the steady-state of unloading; the peak overshoot $\sigma_{p}^{\prime}(\%)$ : the percentage of valley value of air-force exceeding the steady value of unloading (s); the error band $\pm \Delta$ ': the fluctuation of air-force after the system reaches the steady-state of unloading; the steady-state overshoot $\sigma^{\prime}(\%)$ : the percentage of valley steady value exceeding setting value.

The statistical analysis was carried out using SPSS statistics 25 (IBM, USA). The data of the control performance evaluation index from CLAFD and TA were processed by one-way analysis of variance (ANOVA). The significance of differences was determined by Duncan's multiple range test. Normality tests were performed at a significance level of $p \leq 0.05$.

\subsection{Performance analysis of CLAFD technique}

Based on the structure design and software system development discussed above, the CLAFD was developed. In this study, the four function modules of CLAFD were used to analyze its performance.

\subsection{Transient replicability}

The CLAFD system consisted of four functional modules. All the loading signals on the surface of the sample were controlled by the air-force control model, therefore, we took the transient module as the research object and explored the replicability of the CLAFD system.

Figure 5 presents the results in five excitations of samples at different hardness through the CLAFD system. The Figure shows that the peak value, the peak time and the energy required for the five excitations were basically the same regardless of hardness. By statistical analysis on the 5 excitations of the 120 samples, the peak error was $(0.0021 \pm 0.0006) \mathrm{mm}$, the peak time error was $(0.0149 \pm 0.0042) \quad \mathrm{s}$, and the excitation energy error was $(0.0616 \pm 0.0012) \mathrm{mm} \cdot \mathrm{s}$, as shown in Table 1. 


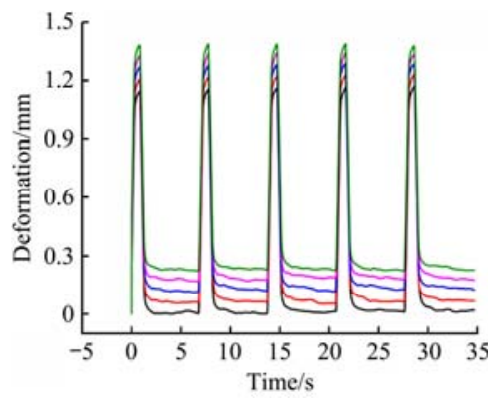

a. $10 \mathrm{HA}$

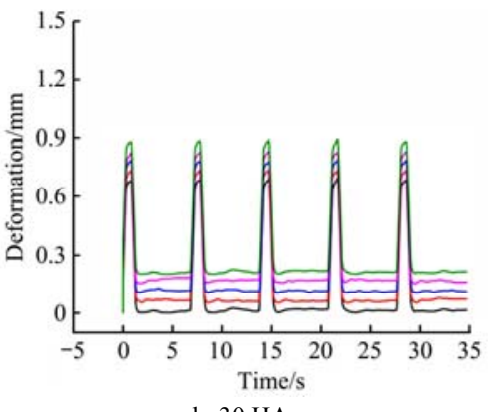

b. $30 \mathrm{HA}$

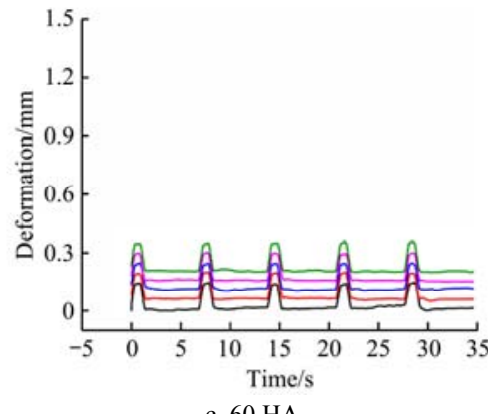

c. $60 \mathrm{HA}$

Figure 5 Replicability of system transient response ( $0.05 \mathrm{~mm}$ deformation offset)

Table 1 Transient repeatability parameters of CLAFD technique

\begin{tabular}{ccc}
\hline Peak error $/ \mathrm{mm}$ & Peak time error/s & Excitation energy error $/ \mathrm{mm} \cdot \mathrm{s}$ \\
\hline $0.0021 \pm 0.0006$ & $0.0149 \pm 0.0042$ & $0.0616 \pm 0.0012$ \\
\hline
\end{tabular}

\subsection{Viscoelastic discrimination by alternating load}

In the study of dynamic mechanical behavior of materials, the most commonly used alternating stress is the sinusoidal stress, which can be expressed as in Equation (2):

$$
\tau(t)=\tau_{0} \sin \omega t
$$

where, $\tau_{0}$ is the amplitude of the stress, $\mathrm{N} ; \omega$ is the angular frequency, $\left({ }^{\circ}\right) / \mathrm{s} ; t$ is the time, $\mathrm{s}$.

Under the action of the sinusoidal alternating stress, the deformation response would change with the different material properties. For the ideal elastomer, since the response of the deformation to the force was transient, the response to the sinusoidal alternating force should also be a sinusoidal function with the same phase, as shown in Equation (3):

$$
\gamma(t)=\gamma_{0} \sin \omega t
$$

where, $\gamma(t)$ is the strain; $\gamma_{0}$ is the strain amplitude.

The rheological behavior of an ideal viscous body obeys Newton's law, that, the stress is proportional to the strain rate, and the proportional coefficient is viscosity. Taking the shear rheology as an example, Newton's law is expressed as:

$$
\tau=\eta \frac{\mathrm{d} \gamma}{\mathrm{d} t}=\eta \dot{\gamma}
$$

where, $\tau$ is the shear stress, $\mathrm{N}, \eta$ is the shear viscosity, $\mathrm{Pa} \cdot \mathrm{s} ; \dot{\gamma}$ is the shear rate, $\mathrm{s}^{-1}$. Equation (3) is substituted into Equation (4):

$$
\gamma(t)=\gamma_{0} \sin \left(\omega t-90^{\circ}\right)
$$

where, $\gamma(t)$ is the strain; $\gamma_{0}$ is the strain amplitude. As observed in Equation (5), the strain of the ideal viscous material would lag behind the stress $90^{\circ}$. Therefore, for a viscoelastic material, the strain would lag the stress by a phase angle $\delta$ and satisfied $0^{\circ}<\delta<90^{\circ}$

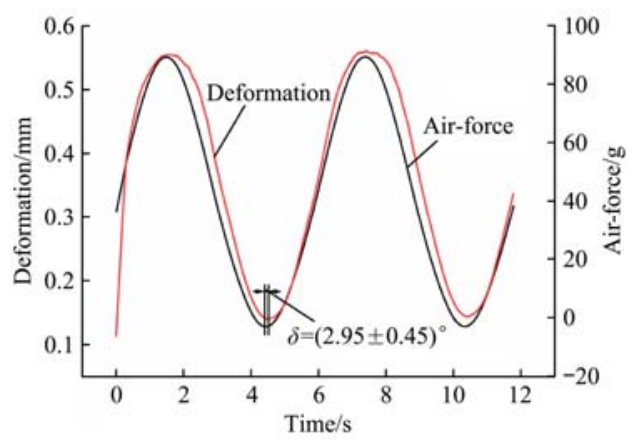

Figure 6 Sinusoidal phase difference of air-force signal and deformation signal
Figure 6 shows that the air-force and deformation of the polyurethane material did not coincide, and the deformation curve had a lagging phase angle $\delta$ with respect to the air-force curve. By statistical calculation, the lagging phase angle was $0^{\circ}<\delta=$ $2.95^{\circ} \pm 0.45^{\circ}<90^{\circ}$. This result indicated that the polyurethane used in this study was a viscoelastic material. The phase angle of the hysteresis was small, which indicated that the polyurethane material exhibited a highly elastic state.

\section{Results and analyses}

\subsection{Comparative analysis between CLAFD and TA on creep-recovery test}

CLAFD system was triggered after the parameters' setting of the creep-recovery test module; the solenoid valve and the electric proportional valve worked successively, and the corresponding program was executed. The surface air-force of the tested sample quickly rose to $40 \mathrm{~g}$ from $0 \mathrm{~g}$. After $60 \mathrm{~s}$ of constant loading, the electrical proportional valve and the solenoid valve were closed, the surface air-force of the sample was rapidly attenuated to $0 \mathrm{~g}$ and remained at $0 \mathrm{~g}$ lasting $60 \mathrm{~s}$ until the end of the test.

Figure $7 \mathrm{a}$ shows that at the initial point, the air-force did not reach the set value in a transient. After a short period of time, the air-force continued to rise, exceeding the set value to reach the peak value, and then the air-force fell back. After repeated adjustments, the air-force stayed near the setting value. The unloading phase was the reverse process of the loading phase. After the constant loading, the system started to unload, and the unloading process was also not completed in an instant, and it took about $0.508 \mathrm{~s}$ as shown in Figure $7 \mathrm{~b}$. In this case, there were differences in the test results produced by the CLAFD and TA. For the TA, when the force reached the unloading steady state, it would continue to drop until it produced an obvious valley value. This valley value could be caused by the existence of the surface viscosity of polythene, when the probe of TA was about to break away from the material (Figure 8a). For the CLAFD, when the air-force dropped to zero, the air-force disappeared, the air-force completely separated from the surface of the sample, and the viscosity of the sample did not work, so there was no obvious valley value generated for the CLAFD.

The creep-recovery test response signals of CLAFD and TA are shown in Figures $7 \mathrm{c}$ and 8b, respectively. During the loading phase, the deformation rapidly increased, it entered the constant force application response phase, and the deformation curve raised slowly. The samples produced significant creep phenomena for both two test methods.

During unloading, the force on the sample was nearly reduced to the steady-state value, the sample deformation was gradual recovery. However, the test results of CLAFD and TA were different. For CLAFD, the sample was continuously 
recovered to the initial position where the deformation became 0 . For TA, the deformation continued to decrease after the deformation exceeded the initial position. The deformation of samples exhibited negative value, and the state was maintained through the end of the test. The surface of the sample was higher than the initial position after TA testing, purportedly, a "failing" state. This "failing" state was due to the contact between the surface and the probe of TA.

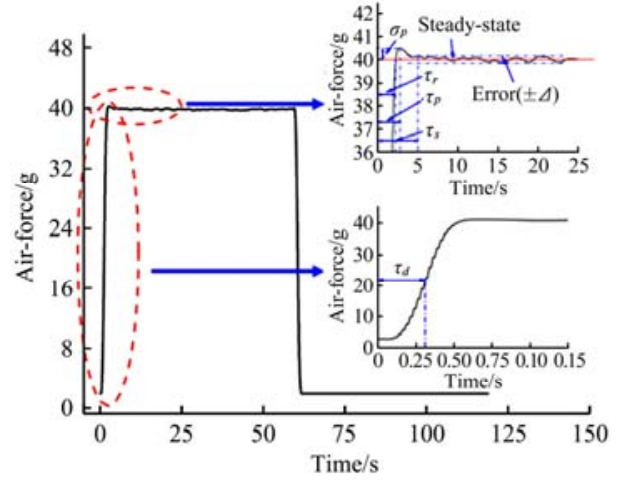

a. Load signal

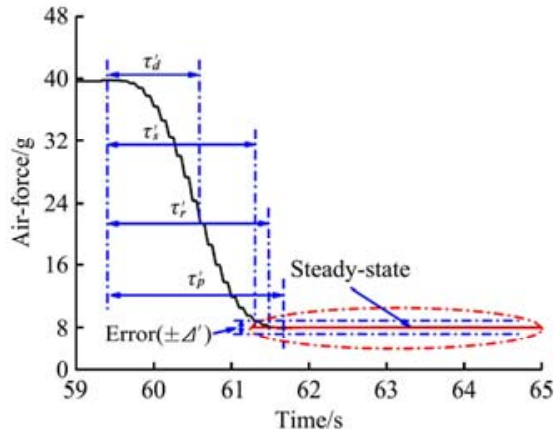

b. Unload stage

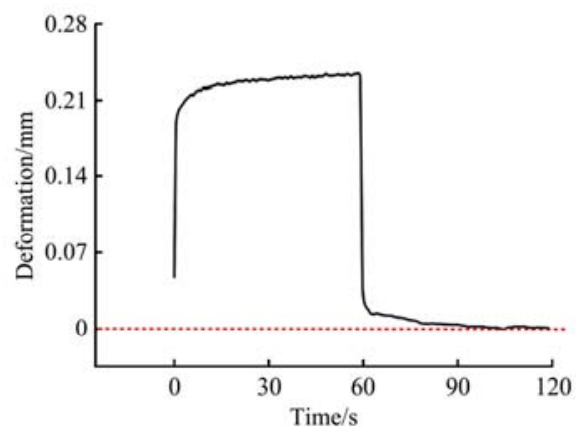

c. Response signal

Figure 7 Creep-recovery property of polymer that was detected by CLAFD

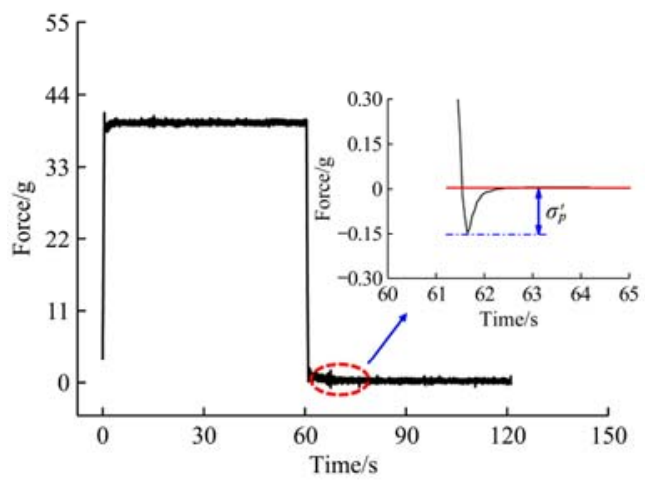

a. Load signal

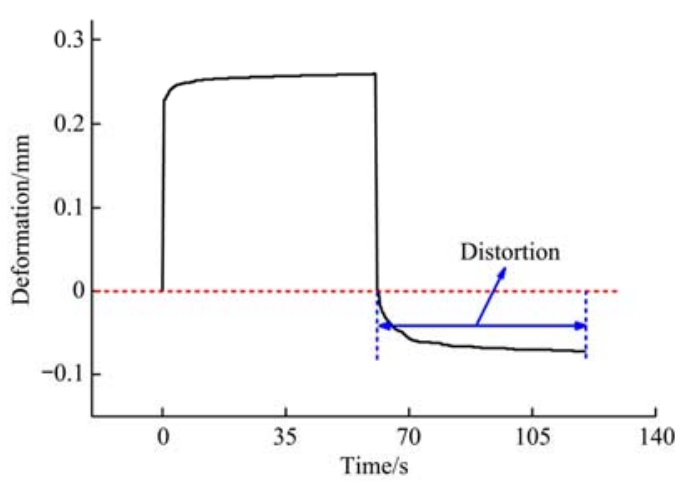

b. Response signal

Figure 8 Creep-recovery property of polymer that was detected by TA

For the system response time of the loading phase (Table 2), the rising time, delay time and peak time of CLAFD were slightly longer than TA. However, there was no significant difference between the two tests. The steady-state adjustment time of the former was significantly shorter than that of the latter. This was because the CLAFD can quickly enter the steady-state region ( $\pm 2 \%$ of the steady-state value) after reaching the peak, while the TA needed a longer time compared with the CLAFD. For the system control accuracy, the three parameters, peak overshoot, steady-state overshoot, and error band of the CLAFD were smaller than the corresponding parameters of the TA. The differences were significant, which indicates that the control precision of CLAFD was significantly better than that of the TA.

For the system response time of the creep-recovery unloading phase (Table 3), the delay time, rise time, peak time, and adjustment time of the CLAFD were shorter than the corresponding parameters of the TA. There were significant differences between the two tests, which showed that the CLAFD reacted faster than the TA. For the relevant indicators of system control accuracy, the values were zero for three parameters of the CLAFD: the peak overshoot, steady-state overshoot and error band. This was significantly smaller than the corresponding parameters of the TA, due to the fact that the solenoid valve and the electromagnetic proportional valve of the CLAFD were shut off immediately after the unloading process started. There was no air-force loading on the surface of the sample. However, the probe of the texture analyzer always contacted the surface of samples during the unloading process in order to acquire the deformation signal. The control accuracy was influenced by sample situation for the TA. Therefore, the performance of CLAFD had obvious advantages in the unloading phase compared to the TA.

Table 2 Compare the creep-recovery properties parameters between the CLAFD and the TA in the loading process

\begin{tabular}{cccccccc}
\hline & $\tau_{d} / \mathrm{s}$ & $\tau_{r} / \mathrm{s}$ & $\tau_{p} / \mathrm{s}$ & $\tau_{s} / \mathrm{s}$ & $\sigma_{p} / \%$ & $\sigma / \%$ \\
\hline CLAFD & $0.323^{\mathrm{a}} \pm 0.005$ & $0.508^{\mathrm{a}} \pm 0.09$ & $0.557^{\mathrm{a}} \pm 0.023$ & $0.343^{\mathrm{a}} \pm 0.031$ & $1.98^{\mathrm{a}} \pm 0.086$ & $0.34^{\mathrm{a}} \pm 0.021$ \\
TA & $0.215^{\mathrm{a}} \pm 0.063$ & $0.412^{\mathrm{a}} \pm 0.078$ & $0.441^{\mathrm{a}} \pm 0.071$ & $0.550^{\mathrm{b}} \pm 0.089$ & $4.485^{\mathrm{b}} \pm 0.935$ & $0.068^{\mathrm{a}} \pm 0.013$ \\
\hline
\end{tabular}

Note: Different letters $(\mathrm{a}, \mathrm{b})$ in the column mean significantly different $(p<0.05)$; Same letters $(\mathrm{a}, \mathrm{a})$ in the column mean non-significant. The same as below.

Table 3 Compare the creep-recovery properties parameters between CLAFD and TA in the unloading process

\begin{tabular}{|c|c|c|c|c|c|c|c|}
\hline & $\tau_{d}^{\prime} / \mathrm{s}$ & $\tau_{r}^{\prime} / \mathrm{s}$ & $\tau_{p}^{\prime} / \mathrm{s}$ & $\tau_{s}^{\prime} / \mathrm{s}$ & $\sigma_{p}^{\prime} / \%$ & $\sigma^{\prime} / \%$ & $\pm \Delta^{\prime} / \mathrm{g}$ \\
\hline CLAFD & $0.195^{\mathrm{a}} \pm 0.010$ & $0.273^{\mathrm{a}} \pm 0.000$ & $0.599^{\mathrm{a}} \pm 0.135$ & $0.380^{\mathrm{a}} \pm 0.039$ & $0.000^{\mathrm{a}} \pm 0.000$ & $0.000^{\mathrm{a}} \pm 0.000$ & $0.000^{\mathrm{a}} \pm 0.000$ \\
\hline TA & $0.204^{\mathrm{a}} \pm 0.029$ & $0.473^{b} \pm 0.056$ & $11.737^{\mathrm{b}} \pm 3.962$ & $7.731^{b} \pm 5.443$ & $2.00^{\mathrm{b}} \pm 0.000$ & $0.557^{\mathrm{b}} \pm 0.053$ & $0.162^{\mathrm{b}} \pm 0.007$ \\
\hline
\end{tabular}




\subsection{Comparative analysis between CLAFD and TA on stress} relaxation test

The stress relaxation test evaluates the force-changing law of the material by keeping the constant deformation of the material. Figure 9 and Figure 10 illustrate the loading signal (Figures 9a and 10a) and response signal (Figures $9 \mathrm{~b}$ and $10 \mathrm{~b}$ ) for the polyurethane stress relaxation test of the CLAFD and TA, respectively. As shown in Figure 9a, similar to the changing rule of the loading signal of the creep-recovery test, the deformation of the polyurethane increased rapidly when the system of the two test methods was triggered. As shown in Table 4, the force value reached the steady-state region ( $\pm 2 \%$ of the steady-state value) after about $0.599 \mathrm{~s}$, and then reached the peak. Partial deformation is maintained around the steady state by the force adjustment. For the response signal, the force loading on the sample was significantly reduced, and the deformation was constant during the $60 \mathrm{~s}$. The deformation rate (the change in deformation of a material with respect to time) of the sample was rapid at first and then it slowed down. This means that the polyurethane material had obvious relaxation and the relaxation was rapid at first and then slowed down. These results indicate that the two methods, CLAFD and TA, could be applied in the detection of stress relaxation properties. For the specific technical parameters of the excitation signal control system of TA, the delay

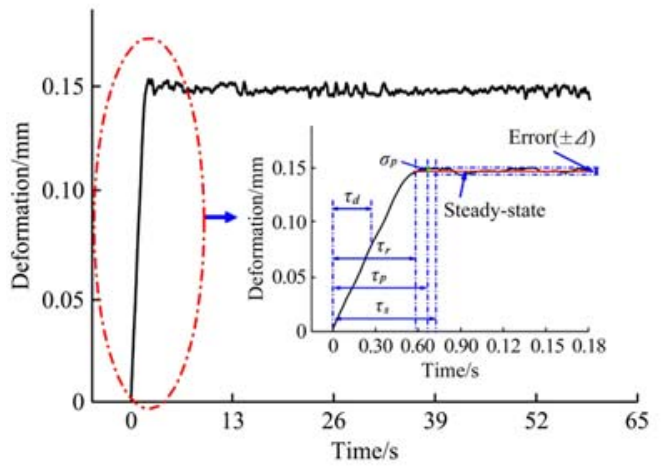

a. Load signal time, rise time, peak time and steady-state adjustment time were significantly shorter than those of CLAFD, which showed that TA was superior to CLAFD in the response speed of the control system. On the control accuracy of the system, the peak overshoot and error band of the TA had obvious advantages over the CLAFD, but nevertheless, the control precision of the CLAFD was better than the TA. Both systems had their own advantages related to control system precision. In general, the TA was superior to CLAFD in reaction speed and control accuracy, because the excitation and response signal of TA was produced by the mechanical structure. When the probe reached the constant deformation setting, the system performed self-locking. Therefore, the error band and peak overshoot of the TA were 0 , but the actual position of probe loading was deviated from the set value due to the structure error of the mechanical system. This could explain why the TA was inferior to CLAFD in the control accuracy of loading. By controlling the opening and closing of the electric proportional valve, CLAFD generated the specific air-force, which generated the required constant deformation. In the process of the relaxation of the sample, the system needed to adjust the air-force constantly in order to obtain deformation. A lot of calculations for the adjustment of air-force would be produced. This may be the reason why the reaction speed of CLAFD was lower than that of TA.

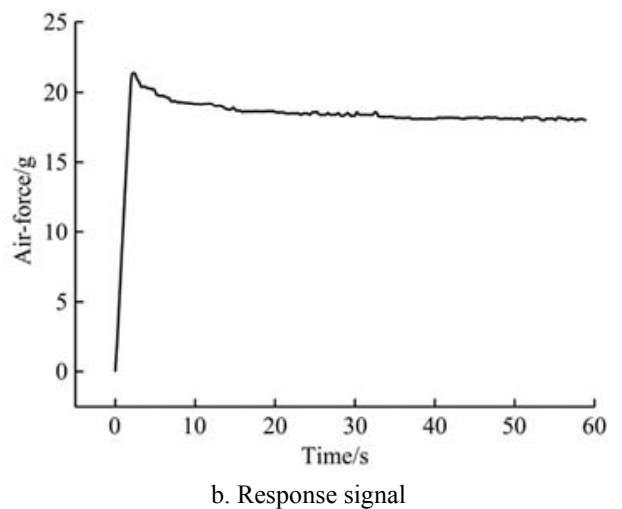

Figure 9 Relaxation property of polymer that was detected by CLAFD technique

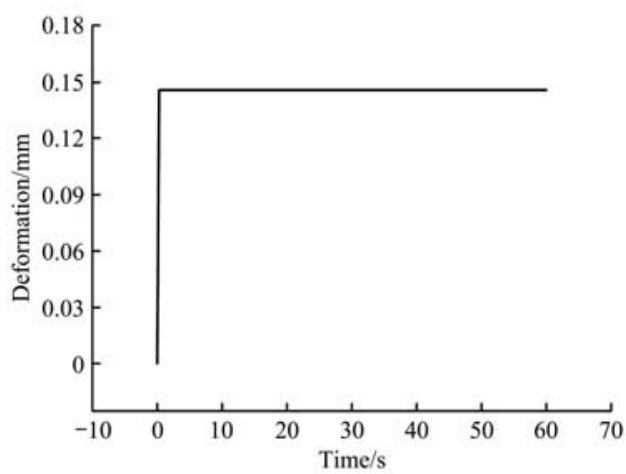

a. Load signal

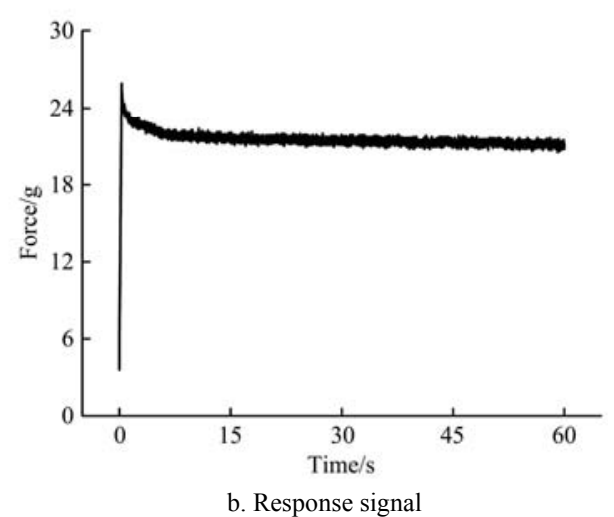

b. Response signal

Figure 10 Relaxation property of polymer that was detected by TA

Table 4 Comparison of the stress relaxation properties parameters between CLAFD and TA

\begin{tabular}{ccccccc}
\hline & $\tau_{d} / \mathrm{s}$ & $\tau_{r} / \mathrm{s}$ & $\tau_{p} / \mathrm{s}$ & $\tau_{s} / \mathrm{s}$ & $\sigma_{p} / \%$ & $\sigma / \%$ \\
\hline CLAFD & $0.320^{\mathrm{a}} \pm 0.091$ & $0.599^{\mathrm{a}} \pm 0.104$ & $0.768^{\mathrm{a}} \pm 0.161$ & $0.826^{\mathrm{a}} \pm 0.125$ & $1.849^{\mathrm{a}} \pm 0.090$ & $0.315^{\mathrm{a}} \pm 0.292$ \\
TA & $0.145^{\mathrm{b}} \pm 0.004$ & $0.289^{\mathrm{b}} \pm 0.008$ & $0.289^{\mathrm{b}} \pm 0.008$ & $0.282^{\mathrm{b}} \pm 0.090$ & $0.000^{\mathrm{b}} \pm 0.000$ & $0.670^{\mathrm{b}} \pm 0.441$ \\
\hline
\end{tabular}

\section{Conclusions}

In this study, a novel polymer rheological property detection system "CLAFD" was developed. Through the design of a small quartz nozzle, the air-force controlling system was created with a practically closed source. This controlled air-force which was produced by the nozzle has good convergence. The deformation information of the polythene was acquired accurately by the laser 
displacement sensor. An artificial neural network algorithm was used to process and analyze 1380 sets of air pressure, distance, and air-force data points. The air-force control model was constructed by the artificial neural network with a correlation coefficient of approximately 1 . The software system of CLAFD was developed with the embedded technology based on the Linux operating system, which realized the high precision control of the polymer rheological property detection. The analysis of peak error, peak time, and excitation energy of polyurethane transient characteristics showed that the system had good replicability. Through the alternating load test of polyurethane material, it was found that there was a lag angle $0^{\circ}<\delta=(2.95 \pm 0.45)^{\circ}<90^{\circ}$ between the deformation and the loading. This indicates that polyurethane was a type of highly elastic viscoelastic medium. Relative to static testing, the CLAFD had obvious advantages in the detection of creep-recovery of polyurethane in comparison to the TA. In the stress relaxation test of polyurethane, although the CLAFD performed well on the individual parameter, the TA was superior to the CLAFD overall. In short, CLAFD is dynamic/static rheological properties detection technology with rich and flexible excitation signals and functions and high control accuracy. Meanwhile, the CLAFD has obvious advantages in the potential application of cross-contamination-free and high-speed detection. It is recommended to promote the development of detection technology for polymer rheological properties with additional research.

\section{Acknowledgements}

The authors gratefully acknowledge the National Natural Science Foundation Project of the People's Republic of China (Grant No. 31571921), the National Science Foundation Project of the United States of America (Grant No. 8018), and the Beijing Natural Science Foundation of the People's Republic of China (Grant No. 6202020).

\section{[References]}

[1] Liang M, Su L P, Li P Z, Shi J T, Yao Z Y, Zhang J, et al. Investigating the rheological properties of carbon nanotubes/polymer composites modified asphalt. Materials (Basel), 2020; 13(18): 4077. doi: 10.3390/ ma13184077.

[2] Hsissou R, Bekhta A, Dagdag O, El Bachiri A, Rafik M, Elharfi A. Rheological properties of composite polymers and hybrid nanocomposites. Heliyon, 2020; 6(6): e04187. doi: 10.1016/j.heliyon.2020.e04187.

[3] Katashima T. Rheological studies on polymer networks with static and dynamic crosslinks. Polym. J., 2021; 53(10): 1073-1082.

[4] Lee J, Kim H. Rheological properties and phase structure of polypropylene/polystyrene/multiwalled carbon nanotube composites. Korea-Aust. Rheol. J., 2020; 32(2): 153-158.

[5] Da Fonsêca J H L, d'Ávila M A. Rheological behavior of carboxymethylcellulose and cellulose nanocrystal aqueous dispersions. Rheol Acta, 2021; 60(9): 497-509.

[6] Mijailovic A S, Qing B, Fortunatoc D, Van K J V. Characterizing viscoelastic mechanical properties of highly compliant polymers and biological tissues using impact indentation. Acta. Biomater, 2018; 71: 388-397.

[7] Cacopardo L, Guazzelli N, Ahluwalia A. Characterizing and engineering biomimetic materials for viscoelastic mechanotransduction studies. Tissue Engineering Part B: Reviews, 2021; Ahead of printd. doi: 10.1089/ ten.TEB.2021.0151.

[8] Zhu C Z, Wang X J, Li Z L, Liu J, Zheng J. Research on static and dynamics mechanical characteristics of flexible bearing in harmonic reducer. Int. J. Adv. Robot Syst., 2020; 17(2): 1729881420919953. doi: 10.1177/1729881420919953.

[9] Feng H, Cui X Y, Li G Y. A stable nodal integration method with strain gradient for static and dynamic analysis of solid mechanics. Engineering Analysis with Boundary Elements, 2016; 62: 78-92.

[10] Tanaka M, Nakahata M, Linke P, Kaufmann S. Stimuli-responsive hydrogels as a model of the dynamic cellular microenvironment. Polym J 2020; 52(8): 861-870.

[11] Prussia S E, Astleford J J, Hewlett B, Hung Y C. Non-destructive firmness measurement device. 1994; Patent No. 5372030, USA.

[12] Hung Y C, Prussia S E, Ezeike G O I. Nondestructive firmness sensing using a laser air-puff detector. Postharvest Biol. Tec., 1999; 16(1): 15-25.

[13] Lee Y S, Owens C M, Meullenet J F. Novel laser air puff and shape profile method for predicting tenderness of Broiler breast meat. Poultry Sci., 2008; 87(7): 1451-1457.

[14] McGlone V A, Jordan R B. Kiwifruit and apricot firmness measurement by the non-contact laser air-puff method. Postharvest Biology and Technology, 2000; 19(1): 47-54.

[15] Morren S, Dyck T V, Mathijs F, Luca S, Cardinaels R, Moldenaers P, et al. Applicability of the food texture puff device for rheological characterization of viscous food products. J. Texture Stud., 2015; 46(2): 94-104.

[16] Long Y, Tang X Y, Wang W J, Peng Y K, Dong X G, Kang X L, et al. A unique method for detecting beef tenderness based on viscoelasticity principle. J. Texture Stud., 2017; 48(5): 433-438.

[17] Li Y L, Wang W J, Long Y, Peng Y K, Li Y Y, Chao K L, et al. A feasibility study of rapid nondestructive detection of total volatile basic nitrogen (TVB-N) content in beef based on airflow and laser ranging technique. Meat Sci., 2018; 145: 367-374.

[18] Li Y L, Tang X Y, Shen Z X, Dong J. Prediction of total volatile basic nitrogen (TVB-N) content of chilled beef for freshness evaluation by using viscoelasticity based on airflow and laser technique. Food Chem., 2019; 87: 26-132.

[19] Lan C, Umezuruike L. Approaches to analysis and modeling texture in fresh and processed foods-a review. Food Eng., 2013; 119(3): 497-507.

[20] Wang Y M, Qing D D. Model predictive control of nonlinear system based on GA-RBP neural network and improved gradient descent method. Complexity, 2021; 3: 6622149. doi:10.1155/2021/6622149.

[21] Wen H, Yan T, Liu Z Q, Chen D L. Integrated neural network model with pre-RBF kernels. Science Progress, 2021; 104(3): 1-18. 